\title{
LOW-COST DGPS ASSISTED AERIAL TRIANGULATION FOR SUB-DECIMETRIC ACCURACY WITH NON-RTK UAVS
}

\author{
F. Ioli ${ }^{1, *}$, L. Pinto ${ }^{1}$, F. Ferrario ${ }^{1}$ \\ ${ }^{1}$ Department of Civil and Environmental Engineering (DICA), Politecnico di Milano - \\ (francesco.ioli, livio.pinto)@polimi.it, francesco4.ferrario@mail.polimi.it
}

Commission II, WG II/1

KEY WORDS: Assisted Aerial Triangulation, DGPS, UAV, direct photogrammetry, flight telemetry.

\begin{abstract}
:
The possibility of equipping UAVs with lightweight GNSS receivers in order to estimate the camera position within a photogrammetric block allows for a reduction of the number of Ground Control Points (GCP), saving time during the field work and decreasing operational costs. Additionally, this makes it possible to build photogrammetric models even in morphologically complex areas or in emergency situations. This work is proposing a non-intrusive and low-cost procedure to retrieve the coordinates of the camera projection centre with decimetric accuracy. The method was designed and tested with the quadcopter DJI Matrice 210 V2 drone equipped with a DJI ZENMUSE X5S camera and an Emlid reach M, a low-cost, single-frequency (L1) GNSS receiver. GNSS observations are post-processed in PPK in order to obtain the UAV trajectory. Synchronization between the camera and the GNSS receiver is achieved by looking at the camera triggering timestamps in flight telemetry data, without requiring an electronic connection between camera and the GNSS that may be troublesome with commercial UAVs. Two surveys were carried out, respectively to calibrate and validate the procedure. The validation test evidenced the possibility of obtaining the coordinates of the camera projection centres with decimetric accuracy. The centre of projections can then be employed for GNSS-assisted aerial triangulation as input of the bundle block adjustment. Provided that at least one GCP is used, it is possible to reach centimetric accuracy on the ground.
\end{abstract}

\section{INTRODUCTION}

In the past few years Unmanned Aerial Vehicles (UAVs) have been widely considered as a valuable tool for applications, such as mapping (Zhang, 2008; Remondino et al., 2011; Nex et al., 2014; Neitzel et al., 2011, Taddia et al., 2020), inspections (Buschinelli et al., 2020; Pinto et al., 2020; Zhang et al., 2017), 3D modelling (Püschel et al., 2008; Remondino et al., 2011; Murtiyoso et al., 2017) and hazard monitoring (Chou et al., 2010; Gonçalves et al., 2015; Giordan et al., 2017). By carrying cameras, UAVs are cost-effective alternatives to traditional manned aerial platforms for performing aerial photogrammetry. In order to solve the Bundle Block Adjustment (BBA) and achieve high geometrical accuracy of photogrammetric blocks, measuring a set of Ground Control Points (GCPs) is traditionally required. This operation is usually time-consuming and sometimes it may also be dangerous. By contrast, it is possible to acquire camera position during each shot by mounting small and lightweight GNSS receivers on board quadcopters or fixed-wing drones and it is a state-of-the-art issue. This allows photogrammetric blocks to be oriented by Assisted Aerial Triangulation (AAT) and, therefore, the number of GCPs to be reduced. Early experiments of this technique on multi-copters (Forlani et al., 2019; Štroner et al., 2020; Forlani et al., 2020) and on fixed-wing (Forlani et al., 2018; Benassi et al., 2017; Chudley et al., 2019) laid the groundwork for the procedure and identified advantages as well as limits and problems. In particular, the pros can be found in the lower cost of the surveys and in the possibility to survey interdicted areas; whereas the cons include the need of a very good calibration of the sensors and the necessity to detect at least some GCPs.

Subsequent studies and improvement in the lightweight GNSS technologies, have made the method competitive with indirect

* Corresponding author photogrammetry by reaching accuracies up to few times the Ground Sample Distance (GSD). Studies such as (Benassi et al., 2017; Forlani et al., 2018, Ekaso et al., 2020) tested GNSSassisted aerial triangulation on a commercial fixed-wing drone equipped with a RTK GNSS receiver. These works report on the accuracy in object space obtained by GNSS-supported orientation of some photogrammetric blocks, all flown according to a flight plan from 30 to $80 \mathrm{~m}$ above ground over a test field. Without GCP, the RTK solution consistently achieves an RMSE of about $2-5 \mathrm{~cm}$ on the horizontal coordinates of checkpoints. In elevation, the RMSE varies from flight to flight, from 2 to $10 \mathrm{~cm}$. Accuracy improves if one or more GCPs are introduced in BBA.

One of the major issues to be solved is the time synchronization between the camera and the GNSS receiver: when the UAVs is flying at around $5 \mathrm{~ms}^{-1}$, a synchronization precision of $\sim 1 / 100 \mathrm{~s}$ is mandatory for achieving decimetric accuracy at the level of the cameras, which may allow for 3D accuracy of $2-5 \mathrm{~cm}$ on the ground. Synchronization is usually obtained by recording an electrical signal from the camera hot-shoe connector, but other synchronization methods, e.g. based on the time-stamping of the trigger pulse sent by the autopilot to the camera, has been explored (Rehak and Skaloud, 2017).

Another aspect that was particularly studied and analysed was related to GCPs. In the above-mentioned studies (Benassi et al., 2017; Forlani et al., 2018) several tests have been carried out comparing the results of indirect photogrammetry, taken as a reference, with those of GNSS-supported photogrammetry and varying the configuration and disposition of GCPs. These experiments demonstrated the impossibility to achieve centimeter accuracies in the absence of GCPs, identifying high errors on check points in this context, usually not compatible with the requirements, in terms of accuracy, of drone photogrammetry. By contrast, the presence of at least one or a 
small set of well distributed GCPs guarantees the joint estimation of camera focal length and camera height with respect to the terrain elevation. This is therefore a necessary condition to be able to adequately bind the block on the ground and significantly reduce errors on check points (Skarlatos et al., 2013; Przybilla et al., 2020; Benassi et al., 2017; Forlani et al., 2018). Moreover, the tests carried out by (Benassi et al., 2017) identified the importance of properly planning the flights by including nadiral and oblique images and several cross-stripes within the survey. All the previous studies confirmed the potential of GNSSassisted aerial triangulation and direct photogrammetry by UAVs, highlighting the critical factors but also many positive aspects, such as the reduction of survey execution time or the possibility of flights in areas or emergency situations.

This study is proposing a low-cost, non-intrusive and easy implementable system to obtain camera projective centres with decimetric accuracy for a non RTK-ready commercial UAV, the quadcopter DJI Matrice 210 V2, which can be used to solve the Bundle Block Adjustment by AAT in order to achieve centimetric accuracy on the ground and to reduce the number of GCPs to be used.

\section{METHODS AND INSTRUMENTS}

\subsection{Assisted Aerial Triangulation procedure}

The proposed method enables the Bundle Block Adjustment (BBA) to be solved by GNSS Assisted Aerial Triangulation (AAT) by providing Antenna Phase Centre (APC) coordinates, at mid-exposure time, and the offset between the GNSS APC and Camera Projection Center (CPC), commonly known as the level arm offset (Ekaso et al., 2017; Benassi et al, 2017).

Usually, the synchronization between the GNSS receiver and the camera is obtained by an electrical signal transmitted through the camera hotshoe connector, typically used to trigger external flash (Chudley et al 2019; Dinkov, 2019) or with external synchronization and triggering module (Ekaso et al., 2017). However, this requires either the use of a high-quality compact or mirrorless camera or an intrusive hardware modification of the camera. Most off-the-shelves UAVs have the camera embedded within the gimbal stabilization system and therefore the access to it may be cumbersome. In this study the synchronization between the camera and the GNSS receiver is searched in the UAV

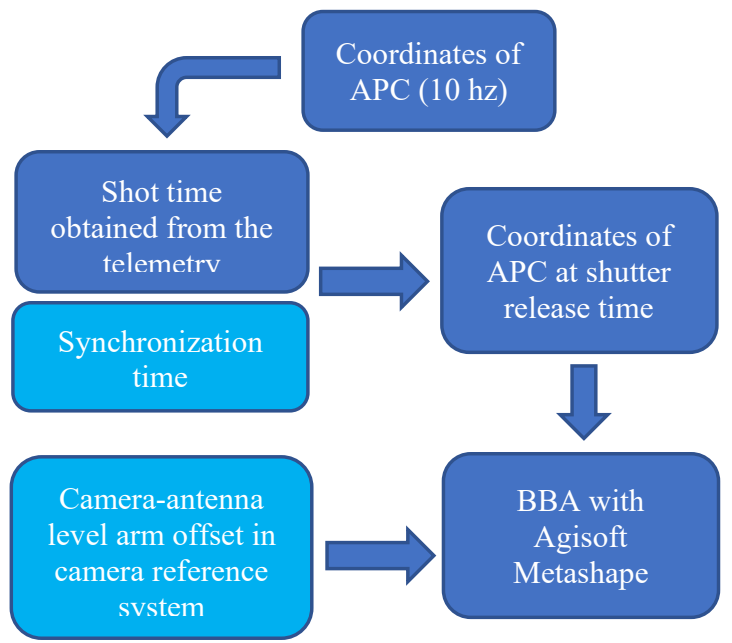

Figure 1. Flowchart of the Assisted Aerial Triangulation procedure. telemetry, recorded with a $200 \mathrm{~Hz}$ sampling rate by the onboard autopilot system. If the flight is controlled automatically by a ground control station software, a label such as "Succeeded Shot" is usually marked in telemetry when the camera shot is trigged by the autopilot. However, it is known that there is a delay between the time at which the camera is triggered and the midexposure time. Synchronization is thus obtained by calibrating this delay. Given the mid-exposure time of each shot, it is possible to interpolate the APC coordinates from the GNSS trajectory. If the position of the GNSS antenna is known, the CPC is determined by applying corrections due to camera-antenna level arm offset. This is determined through a calibration procedure on a test site keeping the camera nadiral: in this way, oblique images that vary the offset value must be excluded during the flight. The complete workflow of the procedure is illustrated in Fig. 1.

The proposed is therefore easily implementable on all commercial off-the-shelves multi-copters, provided that shooting time-stamps are recorded in the flight telemetry and a good quality GNSS trajectory is available.

\subsection{Description of instruments}

For this study, a commercial quadcopter DJI Matrice $210 \mathrm{~V} 2$, not the RTK-ready version, was used. It was equipped with a DJI Zenmuse X5S camera with $20 \mathrm{Mpx} 4 / 3$ " CMOS sensor and electronic shutter, mounted on a 3-axis gimbal. The lens used was a DJI MFT $15 \mathrm{~mm} / 1.7 \mathrm{ASPH}$.

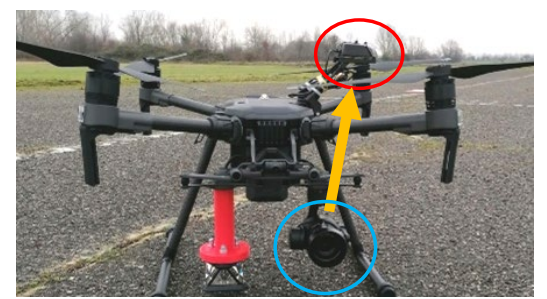

Figure 2. The quadcopter DJI Matrice 210 v2 used for the tests. The blue circle marks the position of the camera, the DJI $\mathrm{X} 5 \mathrm{~S}$, the red circle highlights the GNSS antenna placement.

The yellow arrow denotes the level arm offset between the camera and the antenna.

Regarding the GNSS components, a compact and low-cost single frequency receiver Emlid Reach $M$ with a patch antenna was installed on the UAV (Fig. 1) and powered on from the $5 \mathrm{~V}$ output USB port of Matrice 210, without any other connection with the drone. The receiver was designed to log raw observations from GPS, GLONASS, GALILEO and SBAS constellations. These can be post-processed with a PPK approach with respect to a local master station or in NRTK in order to obtain the APC trajectory during the flight. The commercial software Agisoft Metashape was used to solve the BBA.

\subsection{Test organization and surveys}

The experiment was organized as follows: two surveys were performed at an aeromodelling airfield near Piacenza, Italy $\left(44^{\circ} 58^{\prime} 22^{\prime \prime} \mathrm{N} 9^{\circ} 35^{\prime} 48^{\prime \prime}\right.$ E) respectively on 17/12/2020 and $28 / 01 / 2021$. The test site encloses an area of about $150 \times 100 \mathrm{~m}^{2}$, without any trees nor obstacles. The December flight, named hereafter flight $C$ (where $C$ stands for calibration, Fig.3) was used for calibrating the system. The January survey, flight $V$ was used 


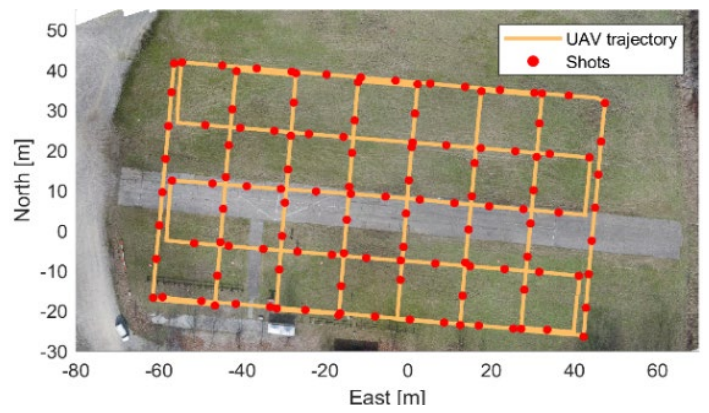

Figure 3. Flight trajectory of Flight $C$, employed for calibrating the system. Red dots denote shot positions.

to validate the method. Both flights were planned with $70 \%$ forward overlap and $60 \%$ transversal overlap and with a doublegrid cross stripes configuration. These were made fly at about 35 $\mathrm{m}$ above ground level, with a GSD of about $0.8 \mathrm{~cm}$. The photogrammetric block in survey $C$ consisted of 5 primary stripes, roughly oriented in E-W directions, and 7 transversal stripes, roughly N-S, producing a total of 111 images. The block in survey $V$ was slightly larger and consisted of $6 \mathrm{E}-\mathrm{W}$ stripes and $8 \mathrm{~N}-\mathrm{S}$ ones, producing a total of 156 images. All the flights were planned and controlled automatically by the GCS software UgCS.

On the ground, 9 home-built targets were materialized for flight $C$, while 10 targets were employed for flight $V$. These were measured with a MultiStation Leica MS60 (Fagandini et al., 2017) in a local reference system with $x$ and $y$ axes in the horizontal plane, directed towards East and North respectively.

\subsection{Post-Processing Kinematic of GNSS observation}

Raw GNSS observations were stored in a RINEX 3.03 file by the Emlid Reach M receiver, mounted on-board the UAV, with a sampling rate of $5 \mathrm{~Hz}$. These were post-processed in PPK with the open source software RTKLIB v.2.3.4b33.

For the calibration flight (flight $C$ ), a geodetic quality GNSS receiver was used as local master station. Its coordinates were determined within ETRF2000 (2008.0) reference frame by means of a static positioning of $\sim 30 \mathrm{~min}$ with respect to 3 permanent stations located in a radius of $\sim 50 \mathrm{~km}$.

For the validation flight $V$, on the other hand, GNSS observations during the flight were processed with respect to a permanent station of the HxGN SmartNet network located $10 \mathrm{~km}$ away from the test site. Again, APC coordinates were obtained in ETRF2000 (2008.0). Both in survey C and V, a Fix-and-Hold approach was used to fix the phase ambiguity and almost all the solution obtained were fixed.

\section{SIMULATIONS}

A series of simulations of a test block were performed using the software CALGE (Pinto et al., 2005) in order to evaluate the impact of Camera Projective Centres (CPC) positional constrains on the block quality. By solving the BBA, the software is able to return the estimation accuracies of a block of images in which pseudo-observation equations on individual parameters (i.e. CPC coordinates, coordinates of self-calibration parameters, coordinates of GCP) are introduced. The simulated block had dimensions of about $120 \mathrm{~m} \times 150 \mathrm{~m}$ of flat terrain and was composed of 170 images arranged on 13 gridded stripes acquired

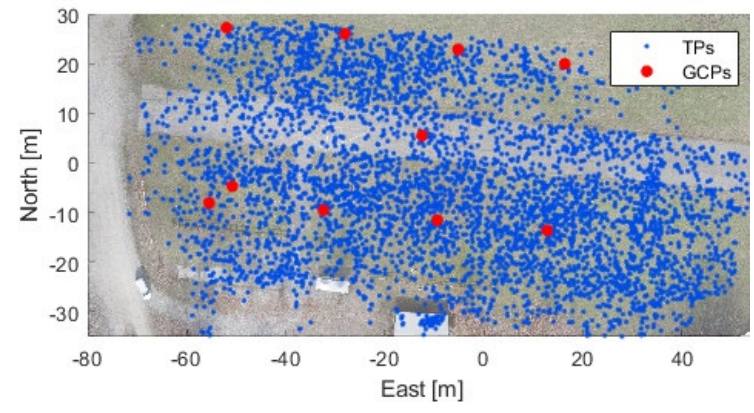

Figure 4. Tie Points (TPs) and GCPs used in the block simulation.

at about 30 m relative altitude with $90-60 \%$ overlapping; 9 GCPs were present on the terrain (Fig. 3). The presence of 4659 tie points was simulated. Regarding the a-priori accuracies of the $\mathrm{CPC}$, in order to evaluate the influence of the uncertainty in estimating the position of the GNSS antenna at the time of shooting (e.g. due to synchronization or interpolation errors) two different planimetric accuracies of \pm 10 or $\pm 15 \mathrm{~cm}$ have been assumed. The accuracy in elevation has been maintained at \pm 5 $\mathrm{cm}$ because the UAV should not change its altitude quickly during a levelled flight. For GCPs, a-priori accuracies of $\pm 2 \mathrm{~cm}$ in the three coordinates were assumed. Accuracies of $\pm 10 \mu \mathrm{m}$ were imposed on the photogrammetric observations.

Six different simulations were performed. First, the traditional case of indirect photogrammetry was simulated: the coordinates of 7 GCPs were constrained. The following 2 simulations were performed without GCPs, varying the a-priori planimetric accuracy of the projective centres. In the following simulations, the number of GCPs was modified. Except in cases 2 and 3 (where the external orientation parameters were fixed), the focal length, the position of the principal point of the camera and parameters B1 and B2 (able to estimate the effects of the rolling shutter) were estimated in auto-calibration. The quality of the results was assessed by analysing the estimation accuracies on the Control Points (CP). The results obtained are presented in Tab. 1.

The results of the simulations allow the following conclusions to be made: the aerial triangulation block with constraints on CPC evidences comparable accuracy to that constrained with GCPs

\begin{tabular}{|c|c|c|c|c|c|c|c|c|}
\cline { 4 - 8 } \multicolumn{2}{c|}{} & $\begin{array}{c}\text { CPC St. Dev. } \\
{[\mathrm{mm}]}\end{array}$ & \multicolumn{2}{c|}{$\begin{array}{c}\text { CPS St. Dev. } \\
{[\mathrm{mm}]}\end{array}$} & $\begin{array}{c}\text { Estimated } \\
\text { auto- } \\
\text { calibration } \\
\text { parameters }\end{array}$ \\
\hline \# & GCP & CP & X, Y & Z & X & Y & Z & c, cx, cy, B1, B2 \\
\hline 1 & 7 & 2 & - & - & 11 & 11 & 13 & c, \\
\hline 2 & 0 & 9 & 100 & 50 & 11 & 12 & 9 & - \\
\hline 3 & 0 & 9 & 150 & 50 & 16 & 16 & 12 & - \\
\hline 4 & 1 & 8 & 150 & 50 & 14 & 15 & 10 & c, cx, cy, B1, B2 \\
\hline 5 & 2 & 7 & 150 & 50 & 12 & 12 & 9 & c, cx, cy, B1, B2 \\
\hline 6 & 5 & 4 & 150 & 50 & 9 & 9 & 7 & c, cx, cy, B1, B2 \\
\hline
\end{tabular}

Table 1. Results in terms of Check Points (CP) accuracies for a series of simulations performed on a test block of 170 images. In the table, X, Y, Z are the coordinates of the 3D world reference system (where $\mathrm{Z}$ is the vertical direction). Regarding the autocalibration parameters: $\mathrm{c}$ is the focal length; $\mathrm{cx}$ and cy are the coordinates of the principal point; B1, B2 are the affinity parameters, according to the Brown's model (Brown, 1971). 
(simulations 1 and 2 or 3 ). The accuracy of the camera projective centres only marginally affects the accuracy of the control points. This is shown by the results of simulations 2 and 3. Simulations 4 and 5 show that the presence of at least 1 GCP allows to estimate some self-calibration parameters (in particular the focal length $c$, the position of the principal point $c x$ and $c y$ and parameters $\mathrm{B} 1$ and $\mathrm{B} 2$ ) avoiding systematic errors in $\mathrm{Z}$ due to errors in the focal length. The simultaneous presence of constraints on the camera projective centres and on the GCPs, guarantees the best precision on the control points, providing at the same time the most robust solution also in terms of estimation of the self-calibration parameters. Regarding the estimation of the self-calibration parameters, it is worth noting that, as mentioned in the previous paragraph, at present the system that has been developed supports only the blocks with nadiral camera attitude. This implies that the internal orientation of the camera must be well estimated. In fact, by limiting the number of ground control points to a minimum, not all the internal orientation parameters result estimable with the BBA.

\section{SYSTEM CALIBRATION}

Calibrating thoroughly the system represents a key issue in order to successfully implement the proposed method. First, determining the Camera Internal Orientation (IO) parameters according to the Brown's model (Brown, 1971) is fundamental to perform TAA, especially when few GCPs are used. Moreover, it is necessary to estimate the eccentricity vector between the CPC and the APC. Finally, the delay between the time at which the camera is triggered and the mid-exposure time has to be estimated.

\subsection{Camera Calibration}

Camera IO calibration in UAVs-based photogrammetry blocks is a critical issue and has been widely discussed (James, Robson $\mathrm{S}$, 2014, Harwin et al., 2015; Gerke, Przybilla, 2016; Cramer et al., 2017). According to (Cramer et al. 2017) and (Benassi et al. 2017), due to the inherent instability of the consumer cameras used with UAVs, a robust self-calibration procedure in which the IO parameters are estimated by solving the BBA is desirable. Moreover, because the DJI Zenmuse X5S embodies an electronic shutter, which is prone to produce rolling shutter effects if the camera shoots while moving, a self-calibration performed on an extensive photogrammetric block was chosen as most suitable approach (Fig. 5a). In fact, this allows for a more robust estimation of the affinity parameters B1 and B2, which may partially compensate the rolling shutter effect. To this end, the nadiral block acquired in flight $\mathrm{C}$ with a cross-stripes configuration was used (see Sec. 2.3 for more detail about the flight). Some of the images within the block were acquired with a small tilt with respect to the nadiral direction (i.e. pitch angles between $5^{\circ}$ and $10^{\circ}$ ), thereby reducing the block deformations due to badly estimated radial distortion parameters K1-K3 (James, Robson, 2014) (Fig. 5a).

Additionally, in order to improve the self-calibration quality, initial values of the IO parameters were computed by performing pre-calibration with 43 convergent cameras and 12 GCPs measured with millimetric accuracy by a the MultiStation Leica MS60 (Fig. 5b).

\subsection{Camera-antenna level arm calibration}

In order to perform AAT in Agisoft Metashpe, one possibility is to directly provide the CPC coordinates as input of BBA.

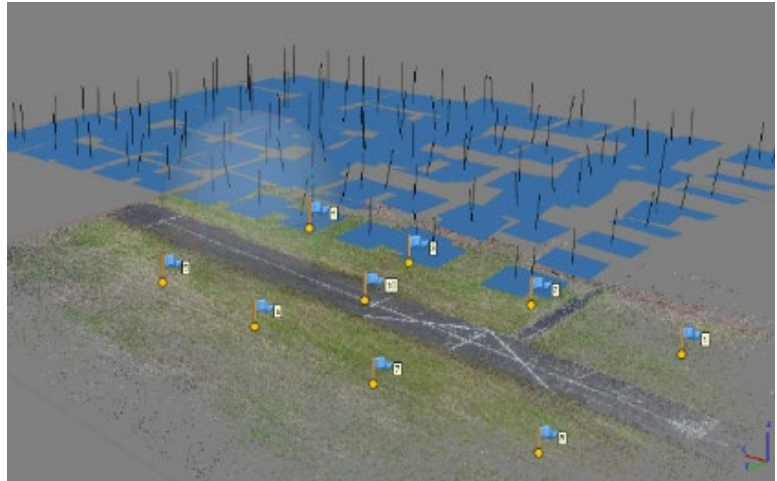

(a)

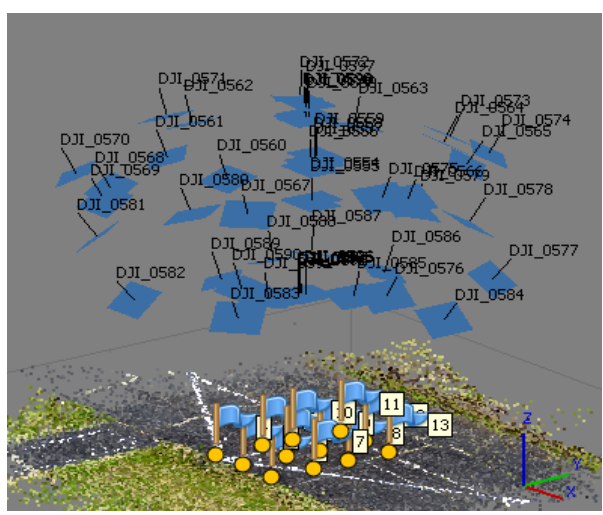

(b)

Figure 5. Photogrammetric blocks used to calibrate the camera: (a) cross-stripes block (flight $C$ ) with 111 (quasi-) nadiral images and 9 GCPs in which self-calibration was performed to estimate IO parameters; (b) calibration site with strongly convergent acquisition geometry used to compute initial values of IO parameters.

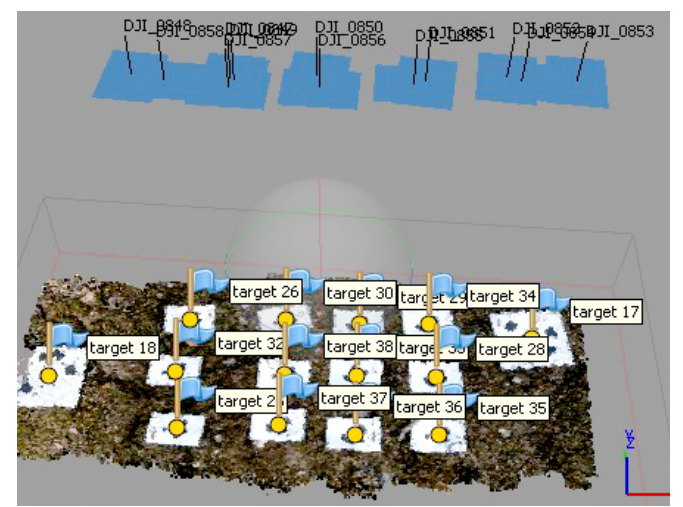

Figure 6. Photogrammetric block used to calibrate the level arm offset between the camera and GNSS antenna. The 14 GCPs used to orient the images are marked with blue flags.

Alternatively, APC coordinates and the offset between the CPC and APC (hereafter named as level arm offset $L$ ) determined in the camera reference system may be supplied. The second procedure was chosen for this work.

In order to calibrate the lever arm vector, a procedure similar to that used by (Forlani et al., 2013) was followed: a small 
photogrammetric block was set up, while recording at the same time GNSS observations with the Emlid receiver. These were post-processed following the procedure described in Sec. 2.4. The photogrammetric block consisted of 12 nadiral camera stations, with different heading direction (i.e., 6 roughly heading north and 6 rotated by $90^{\circ}$ around the $\mathrm{Z}$ axis) (Fig. 6). 14 GCPs were measured with millimetric accuracy with the MultiStation Leica MS60 and rototranslated into the ETRF2000 reference system, the same as for GNSS observations. For each camera station, the UAV and camera were held in the same position for about $30 \mathrm{~s}$, resulting in $\sim 150$ GNSS observations each in order to obtain the APC. Level arm offset in the world reference for the photo $i$ was computed as

$$
L_{i}^{w}=C P C_{i}^{w}-A P C_{i}^{w}
$$

where the superscript ${ }^{w}$ denotes the world reference system.

For each camera, $L_{i}^{w}$ was then rotated into the camera reference system (i.e. $\mathrm{Y}$ towards camera top, $\mathrm{Z}$ opposite to the viewing direction and $X$ completing the right-handed space, Fig. 7) by means of the external orientation angles of each camera, estimated within the photogrammetric block. Level arm offset in the camera reference system $L^{\text {cam }}$ was estimated as the average over all the cameras of $L_{i}^{c a m}$ and its standard deviation can be considered as the estimation error. As shown in Tab. 2, subcentimetric accuracies in planimetry and centimetric accuracy in altitude were achieved by calibrating the level arm offset $L^{\text {cam }}$, within the camera reference system.

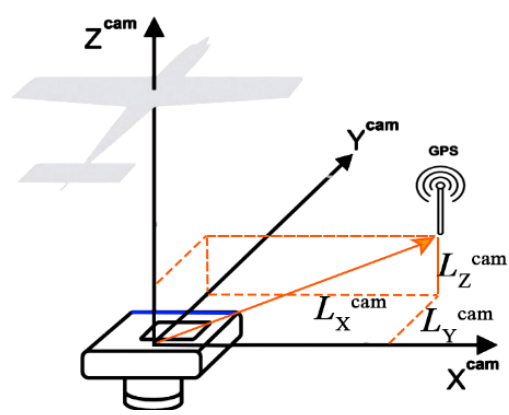

Figure 7. Camera reference system, as defined by Agisoft Metashape (the blu line on the camera denotes the camera top, which points towards the flying direction). The cameraantenna level arm offset $L^{\text {cam }}$ is represented with the orange arrow. Image adapted from Agisoft Metashape User Manual (Agisoft, 2021)

\begin{tabular}{cccc}
\hline & $\boldsymbol{L}_{X}^{\text {cam }}[\boldsymbol{m}]$ & $\boldsymbol{L}_{Y}^{\text {cam }}[\boldsymbol{m}]$ & $\boldsymbol{L}_{Z}^{\text {cam }}[\boldsymbol{m}]$ \\
\hline Mean & -0.063 & -0.134 & 0.310 \\
Std & 0.007 & 0.005 & 0.010
\end{tabular}

Table 2. Mean and standard deviation of the estimated of the estimated camera-antenna level arm vector in the camera reference system.

It should be noted that during calibration both the camera and the UAV were kept levelled as much as possible, i.e. with UAV roll and pitch angles smaller than $2^{\circ}$, camera kept nadiral by the gimbal and with the sensor top looking front, towards the UAV nose. This specific camera reference system is hereafter labelled as Camera Levelled Reference System (CLRS). Therefore, these will also be the requirements to be fulfilled in order to use the estimated $L^{\text {cam }}$ as input of BBA. Nevertheless, it is not feasible to maintain a perfectly levelled condition during flights (e.g. due to wind or to UAV acceleration). Yet, the rotation of the UAV up to $10^{\circ}$ in roll and pitch around the camera (kept nadiral by the gimbal) may be acceptable because it mostly causes planimetric errors up to $5 \mathrm{~cm}$, which is compatible with the aimed CPC estimation accuracy.

\subsection{GNSS-camera synchronization}

As previously mentioned, there is a delay $d t$ between the time at which the camera is triggered and the actual mid-exposure time (known as shutter delay) that must be calibrated (Rehak and Skaloud, 2017).

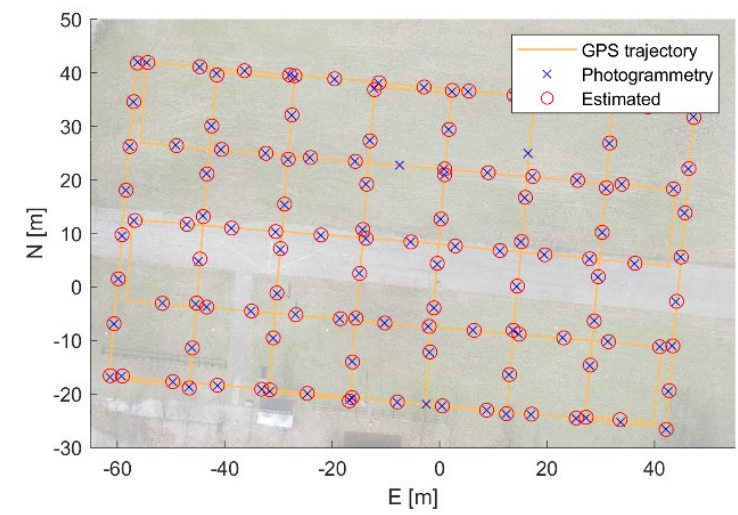

Figure 8. Comparison between the APC position interpolated from the GNSS trajectory (red circle) and the photogrammetric $\boldsymbol{C P C}-\boldsymbol{L}^{\boldsymbol{w}}$ plotted after the synchronization.

In order to estimate $d t$, the photogrammetric block acquired during survey $\mathrm{C}$ was employed to estimate cameras EO. 5 out of 11 targets were used as GCPs, while the remaining as CPs (onground RMSE on GCPs of $0.6 \mathrm{~cm}$ and on CPs of $1.1 \mathrm{~cm}$ ). Cameras IO was not estimated with self-calibration, but fixed to the that estimated, as described in Sec. 4.1. After having obtained the triggering time from flight telemetry, APC coordinates were interpolated from the GNSS trajectory by cubic interpolation. In order to be directly comparable with the photogrammetric CPC, APC coordinates were corrected by the eccentricity vector $L^{w}$, obtained by rotating $L^{\text {cam }}$ into the world reference system by means of the photogrammetric EO angles of each camera. The shutter delay $d t$ was then estimated as delay along the trajectory minimizing the overall differences between the photogrammetric $C P C-L^{w}$ and those estimated by interpolating the GNSS trajectory (Fig. 8).

A $d t$ equal to $0.42 \pm 0.03 \mathrm{~s}$ was estimated. This is in line with the values of shutter delay estimated by (Rehak and Skaloud, 2017), who determined a delay ranging from $0.406 \mathrm{~s}$ to $0.486 \mathrm{~s}$, with a mean value of $0.0433 \mathrm{~s}$. The estimation uncertainty of $0.03 \mathrm{~s}$ is mainly due to the random component of the shutter delay which can be hardly estimate. If UAV speed is smaller than $5 \mathrm{~m} / \mathrm{s}$, synchronization errors of 0.02-0.04 $\mathrm{s}$ may result in errors up to $0.15-0.20 \mathrm{~m}$ in the CPC estimates, which is not suitable for direct photogrammetry, but may be enough to perform AAT.

\begin{tabular}{cccc}
\hline & $\mathbf{E}[\mathbf{m}]$ & $\mathbf{N}[\mathbf{m}]$ & $\mathbf{U}[\mathbf{m}]$ \\
\hline Mean & 0.069 & 0.041 & 0.054 \\
Std & 0.038 & 0.084 & 0.040 \\
RMS & 0.079 & 0.093 & 0.067 \\
\hline
\end{tabular}

Table 3. Statistics of the differences between the photogrammetric $\mathrm{CPC}$ and the coordinates of $\mathrm{APC}+L^{w}$ after synchronization 


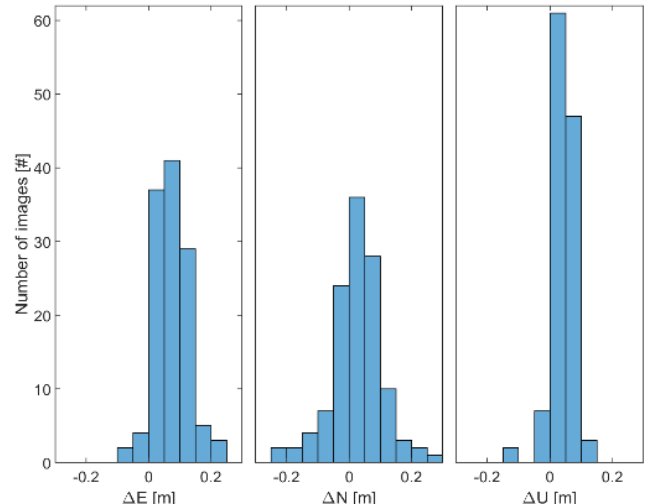

Figure 9. Barplots of the differences between the photogrammetric $\mathrm{CPC}$ and the coordinates of $\mathrm{APC}+L^{w}$ after synchronization.

Performing the interpolation of the GNSS trajectory by considering the estimated value of shutter delay $d t$, differences between photogrammetric $\mathrm{CPC}$ and $\mathrm{APC}+\mathrm{L}^{\mathrm{w}}$ with $\mathrm{RMSE}$ smaller than $0.1 \mathrm{~m}$ were obtained (Tab. 3 and Fig. 9).

\section{VALIDATION ON AN INDIPENDENT DATASET}

The proposed method for performing AAT was assessed by using an independent dataset, called flight $V$, acquired at the same test site as for the system calibration. Flight $V$ was composed of 156 nadiral photos, acquired with a cross-stripes geometry, and 10 targets, used either as GCPs or CPs and measured with subcentimetric accuracy with a Multistation Leica MS60. Other characteristics of the flight are described in Sec. 2.3,

To perform AAT, the full procedure described in Sec. 2.1 was followed: first, the shooting time of each photo was extracted from the telemetry and corrected by the shutter delay; the GNSS trajectory was interpolated to obtain the APC coordinates for each camera. These were used as input of the BBA, in combination with the camera-antenna level arm offset determined within the Camera Levelled Reference System, $L^{\text {cam }}$. The quality of the photogrammetric block was assessed as onground reprojection error on the basis of the CPs only (i.e. the targets not used as GCP in BBA). Several configurations named with letters from $A$ to $G$ (Tab. 3) were tested, varying the acquisition geometry and the number of GCPs.
In each block, the $\mathrm{CPC}$ coordinates were provided as input in the BBA coupled with their a-priori variance, according to the results obtained during the calibration process (see Sec. 4.3). For the images taken within the stripes, when both the UAV and the camera were levelled, a standard deviation of the CPC equal to $0.1 \mathrm{~m}$ in planimetry and $0.05 \mathrm{~m}$ in altitude were assumed (the larger planimetric uncertainty is mainly due to synchronization errors). For border images, when the UAV was accelerating or decelerating and thus the uncertainty in the level arm vector is larger, standard deviations of the CPCs equal to $0.3 \mathrm{~m}$ and $0.15 \mathrm{~m}$ were assumed respectively in planimetry and in altitude.

Block A was oriented by traditional photogrammetry (method labelled as TRAD in Tab. 3), without any information concerning neither APC nor CPC but using 6 GCPs, well distributed over the study area. All the cameras were employed in order to maintain a cross-stripes (called as grid acquisition geometry). Because of the nadiral acquisition geometry, IO parameters modelling the radial $(\mathrm{K} 1, \mathrm{~K} 2, \mathrm{~K} 3)$ and tangential $(\mathrm{P} 1, \mathrm{P} 2)$ lens distortion were fixed to those estimated during calibration in order to mitigate the doming distortion of the photogrammetric model (James and Robson, 2014). Among the IO parameters, these are also the most stable in time. On the other hand, according to the simulation described in Sec. 3, c, cx, cy and B1, B2 may be estimated by solving the BBA if at least one GCP is employed. They were therefore estimated by self-calibration. The 4 CPs denoted subcentimetric model errors in all the direction, comparable to a half of the GSD (i.e., $\sim 0.8 \mathrm{~cm}$ ) in planimetry and slightly higher in height. Hence, Block A is considered as the reference against which all the other configurations can be compared. All the results are summarized in Tab. 4.

Blocks from B to D were oriented by GNSS AAT and a crossstripes acquisition geometry was kept. First, in Block B, only 3 GCPs were used. Again, IO parameters c, cx, cy, B1, B2 only were estimated by self-calibration. Errors on the $7 \mathrm{CPs}$ are comparable to those of Block A, underlying that the reduction of the GCPs is well compensated by the introduction of the APC as input of BBA, without worsening the block accuracy. With the reduction of the number of GCP to one at the centre of the study area (Block C), but with leaving all the other parameters unchanged, the model accuracy slightly increases. Yet, it is never larger than 2 times the GSD.

If no GCP is used, as in Block D, IO parameters can not be estimated by self-calibration, but all of them must be fixed in order not to let the solution diverge and obtain biased results. An accurate camera pre-calibration is therefore required. Without

\begin{tabular}{|c|c|c|c|c|c|c|c|c|c|}
\hline Name & Method & $\begin{array}{l}\text { Acquisition } \\
\text { Geometry }\end{array}$ & $\mathrm{GCP} / \mathrm{CP}$ & $\begin{array}{l}\text { IO params } \\
\text { estimated }\end{array}$ & $\begin{array}{c}\text { Long / Transv } \\
\text { Overlap [\%] }\end{array}$ & $\begin{array}{c}\mathrm{E} \\
{[\mathrm{m}]}\end{array}$ & $\begin{array}{c}\mathrm{N} \\
{[\mathrm{m}]}\end{array}$ & $\begin{array}{c}\mathrm{U} \\
{[\mathrm{m}]}\end{array}$ & $\begin{array}{l}\text { RMSE } \\
{[\mathrm{m}]}\end{array}$ \\
\hline A & TRAD & Grid acquisition & $6 / 4$ & $\begin{array}{c}\mathrm{c}, \mathrm{cx}, \mathrm{cy} \\
\mathrm{B} 1, \mathrm{~B} 2\end{array}$ & $70 / 60$ & 0.005 & 0.004 & 0.010 & 0.012 \\
\hline B & AAT & Grid acquisition & $3 / 7$ & $\begin{array}{l}\mathrm{c}, \mathrm{cx}, \mathrm{cy} \\
\mathrm{B} 1, \mathrm{~B} 2\end{array}$ & $70 / 60$ & 0.006 & 0.004 & 0.013 & 0.014 \\
\hline $\mathrm{C}$ & AAT & Grid acquisition & $1 / 9$ & $\begin{array}{c}\mathrm{c}, \mathrm{cx}, \mathrm{cy} \\
\mathrm{B} 1, \mathrm{~B} 2\end{array}$ & $70 / 60$ & 0.011 & 0.016 & 0.012 & 0.023 \\
\hline $\mathrm{D}$ & AAT & Grid acquisition & $0 / 10$ & none & $70 / 60$ & 0.035 & 0.016 & 0.010 & 0.040 \\
\hline $\mathrm{E}$ & AAT & $6 \mathrm{E}-\mathrm{W}$ stripes only & $1 / 9$ & none & $70 / 60$ & 0.012 & 0.013 & 0.013 & 0.022 \\
\hline $\mathrm{F}$ & AAT & 3 E-W stripes only & $1 / 9$ & none & $70 / 20$ & 0.030 & 0.035 & 0.047 & 0.065 \\
\hline G & AAT & $\begin{array}{l}3 \text { E-W stripes with } \\
2 \mathrm{~N}-\mathrm{S} \text { stripes only }\end{array}$ & $1 / 9$ & none & $70 / 20$ & 0.022 & 0.022 & 0.021 & 0.038 \\
\hline
\end{tabular}

Table 4. Results of the validation of the AAT procedure on the validation flight for the different block configurations. 


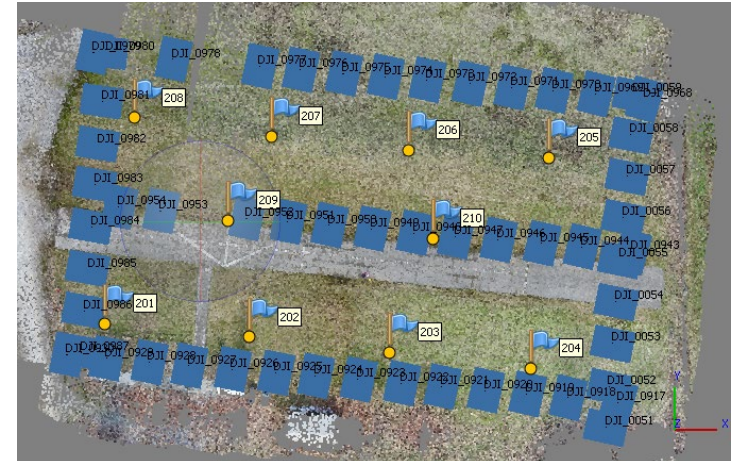

Figure 9. Acquisition geometry of Block G: just 3 E-W stripes were used (with a transversal overlap of $20 \%$ ), but 2 $\mathrm{N}-\mathrm{S}$ stripes were used to constrain the block geometry.

any GCP, CP errors are 2-3 times larger than those obtained with one GCP (Block C), but still the model error is smaller than $5 \mathrm{~cm}$ in all the directions. This result reveals that with GNSS assisted aerial triangulation it is possible to obtain sub-decimetric accuracy, even if the precision of CPC coordinates (or APC plus the level harm) used as input of BBA is one order of magnitude larger (i.e. $15-20 \mathrm{~cm}$ ). Since no self-calibration can be carried out, camera pre-calibration plays a crucial role and is fundamental to provide estimates of the IO parameters which are very close to the actual values.

Additionally, 3 blocks with stripes in one direction only (Blocks from $E$ to $G$ ) were solved in order to assess the performance of AAT with weaker acquisition geometry, compared to the crossstripes gridded flights. In all three cases, only one GCP in the middle of the study area was used. In Block E, where only 6 E$\mathrm{W}$ stripes were used, but high longitudinal and transversal overlaps were kept (respectively $70 \%$ and $60 \%$ ), the results were comparable to those of Block $\mathrm{C}$, obtained with the grid acquisition. However, no self-calibration was carried out in order to avoid mis-estimation of the IO parameters due to poor constrains of the block geometry. In fact, by estimating c, cx, cy, $\mathrm{B} 1$ and $\mathrm{B} 2$ within BBA, the CP reprojection error was almost 3 times larger and the estimated value of focal length $\mathrm{c}$ was significantly different that the true one.

In Block F, the transversal overlap was reduced by removing 3 E-W stripes leading to an extremely poor acquisition geometry (i.e. the minimum requirement to be able to build a photogrammetric model). In this case, the error on the CPs increases significantly, reaching up to 6 times the GSD. However, adding just two N-W stripes (Block G, Fig. 9), drastically improve the BBA solution, bringing the errors back to 2-3 times the GSD.

\section{CONCLUSIONS}

The purpose of this work was to derive the trajectory of the DJI Matrice 210 V2 in order to use its position for assisted aerial triangulation. This was achieved with the integration of a single frequency Emlid GNSS receiver and a calibration procedure. In particular, having aimed to follow a procedure based on the use of the UAV telemetry data (recorded during an automatic flight), it was necessary to calculate the time offset that could correct the time obtained from the triggering time-stamps recorded in the telemetry in order to determine the effective half exposure time of the camera shutter release.
Due to the errors cumulated during the workflow (especially due to the uncertainties in the shooting time synchronization and trajectory interpolation) the position of the CPC at the shooting time is determined with an accuracy of about one decimetre. Nevertheless, even if the accuracy is not at the centimetre level, but provided that it is coupled with the proper a-priori variance, information about the CPC position can be validly used in aerial triangulation to improve the model quality and drastically decrease the number of GCPs to be used.

The tests carried out evidenced that at least one GCP is necessary to estimate some calibration parameters of the camera (in particular the focal length) which, otherwise, could generate unwanted distortion in the model. The results obtained from the validation tests allows to quantify the final model accuracy in 1-5 cm, which corresponds to 1-6 GSD. These outcomes confirmed those obtained in a series of simulations carried out preliminarily on a similar area to that used in the test.

Currently the main limitation of the method is its applicability only to nadiral (or tilted, but known attitude) shots, because the level arm offset is determined with an "ad hoc" calibration procedure with a specific camera attitude. However, the 3-axis gimbal mounted on the DJI Matrice 210 V2 allows for angular variations of the camera with respect to the UAV which can be hardly predicted (e.g., due to wind gusts or accelerations). In this regard, a new procedure is already being tested to exploit the angular information stored in the telemetry to compute the level arm offset for each image, in the object reference system, able to correct the position of the APC and consequently directly determine the CPC.

Regarding the UAV trajectory, the use of a dual-frequency GNSS receiver is at an advanced stage of experimentation, which will reduce time needed to fix the phase ambiguity making the whole procedure more flexible.

\section{REFERENCES}

Agisoft, 2021. Agisoft Metashape User Manual - Professional Edition, Version 1.7. https://www.agisoft.com/pdf/metashapepro_1_7_en.pdf

Benassi, F., Dall'Asta, E., Diotri, F., Forlani, G., Morra di Cella, U., Roncella, R., Santise. M., 2017. Testing accuracy and repeatability of UAV blocks oriented with GNSS-supported aerial triangulation, Remote Sensing, N. 9 (2), 172.

Brown, D. C., 1971. Close-Range Camera Calibration. Photogramm Eng, 37, 855-66.

Buschinelli, P., Salazar, J. D., Regner, D., Oliveira, D., Machado, M., Marcellino, G., Sales, D. C., Santos, J. M., Marinho C. A., Stemmer, M. R., Pinto, T. C., 2020. Targetless photogrammetry network simulation for inspection planning in oil and gas industry. ISPRS Ann. Photogramm., Remote Sens. Spatial Inf. Sci., 1, 285-291.

Chou, T. Y., Yeh, M. L., Chen, Y. C., Chen, Y. H., 2010. Disaster monitoring and management by the unmanned aerial vehicle technology. In: Wagner W., Székely, B. (eds.): ISPRS TC VII Symposium - 100 Years ISPRS, Vienna, Austria, July 5-7, 2010, IAPRS, Vol. XXXVIII, Part 7B, 137-142.

Chudley, T. R., Christoffersen, P., Doyle, S. H., Abellan, A., Snooke, N., 2019. High-accuracy UAV photogrammetry of ice 
sheet dynamics with no ground control. The Cryosphere, 13(3), 955-968.

Cramer M., Przybilla H.J., Zurhorst A., 2017. UAV cameras: Overview and geometric calibration benchmark. Int Arch Photogramm Remote Sens Spat Inf Sci - ISPRS Arch. 42(2) 8592.

Ekaso, D., Nex, F., Kerle, N., 2020. Accuracy assessment of realtime kinematics (RTK) measurements on unmanned aerial vehicles (UAV) for direct geo-referencing. Geo-spatial information science, 23(2), 165-181.

Fagandini, R., Federici, B., Ferrando, I., Gagliolo, S., Pagliari, D., Passoni, D., Pinto, L., Rossi, L., Sguerso, D. (2017). Evaluation of the laser response of Leica Nova multistation MS60 for 3D modelling and structural monitoring. International Conference on Computational Science and Its Applications (pp. 93-104).

Forlani, G., Dall'Asta, E., Diotri, F., Morra di Cella, U., Roncella, R., Santise, M., 2018. Quality assessment of DSMs produced from UAV flights georeferenced with on-board RTK positioning. Remote Sensing, 10(2), 311.

Forlani, G., Diotri, F., Morra di Cella, U., Roncella, R., 2019. Indirect UAV strip georeferencing by on-board GNSS data under poor satellite coverage. Remote sensing, 11(15), 1765.

Forlani, G., Diotri, F., Morra di Cella, U., Roncella, R., 2020. UAV block georeferencing and control by on-board GNSS data. Int. Arch. Photogramm., Remote Sens. Spatial Inf. Sci., XLIIIB2, 9-16.

Forlani, G., Pinto, L., Roncella, R., Pagliari, D., 2013, Terrestrial Photogrammetry without Ground Control Points, Earth Sci Inform, $71-81$

Gerke, M., Przybilla, H.J., 2016. Accuracy Analysis of Photogrammetric UAV Image Blocks: Influence of On board RTK GNSS and Cross Flight Patterns. Photogramm. Fernerkund. Geoinf., 1, 17-30.

Giordan, D., Manconi, A., Remondino, F., Nex, F., 2017. Use of unmanned aerial vehicles in monitoring application and management of natural hazards, Geomatics, Natural Hazards and Risk, 8:1, 1-4.

Gonçalves, J. A., Henriques, R., 2015. UAV photogrammetry for topographic monitoring of coastal areas. ISPRS Journal of Photogrammetry and Remote Sensing, 104, 101-111.

Harwin, S., Lucieer, A., Osborn, J., 2015. The Impact of the Calibration Method on the Accuracy of Point Clouds Derived Using Unmanned Aerial Vehicle Multi-View Stereopsis. Remote Sens. 7(9)

James, M.R., Robson, S., 2014. Mitigating systematic error in topographic models derived from UAV and ground-based image networks, Earth Surf Process Landforms, 39(10)1413-20.

Murtiyoso, A., Koehl, M., Grussenmeyer, P., Freville, T., 2017. Acquisition and processing protocols for UAV images: 3D modeling of historical buildings using photogrammetry. ISPRS Ann. Photogramm., Remote Sens. Spatial Inf. Sci., IV-2/W2.
Neitzel, F., Klonowski, J., 2011. Mobile 3D mapping with a lowcost UAV system. Int. Arch. Photogramm., Remote Sens. Spat. Inf. Sci, XXXVIII-1/C2, 1-6.

Nex, F., Remondino, F., 2014. UAV for 3D mapping applications: a review. Applied geomatics, 6(1), 1-15.

Pinto, L., Forlani, G., Passoni, D. (2005). Experimental tests on the benefits of a more rigorous model in IMU/GPS system calibration. Int. Arch. Photogramm., Remote Sens. Spatial Inf. Sci. Proceedings of XXth ISPRS Congress. Istanbul, Turkey.

Pinto, L., Bianchini, F., Nova, V., Passoni, D., 2020. Low-cost UAS photogrammetry for road infrastructures' inspection. Int. Arch. Photogramm., Remote Sens. Spatial Inf. Sci., XLIII-B2, 1145-1150.

Przybilla, H.-J., Bäumker, M., Luhmann, T., Hastedt, H., Eilers, M., 2020. Interaction between direct georeferencing, control point configuration and camera self-calibration for RTK-based UAV photogrammetry. Int. Arch. Photogramm., Remote Sens. Spatial Inf. Sci. XLIII-B1, 485-492.

Püschel, H., Sauerbier, M., Eisenbeiss, H., 2008. A 3D model of Castle Landenberg $(\mathrm{CH})$ from combined photogrammetric processing of terrestrial and UAV based images. Int. Arch. Photogramm., Remote Sens. Spatial Inf. Sci., XXXVII. Part B6b, pp. 93-98.

Rehak, M., and J. Skaloud. 2017. Time Synchronization of Consumer Cameras on Micro Aerial Vehicles. ISPRS Journal of Photogrammetry and Remote Sensing 123, 114-123.

Remondino, F., Barazzetti, L., Nex, F., Scaioni, M., Sarazzi, D., 2011. UAV photogrammetry for mapping and 3D modelingcurrent status and future perspectives. Int. Arch. Photogramm., Remote Sens. Spatial Inf. Sci., XXXVIII-1/C22, 25-31.

Skarlatos, D., Procopiou, E., Stavrou, G., Gregoriou, M., 2013. Accuracy assessment of minimum control points for UAV photography and georeferencing. First international conference on remote sensing and geoinformation of the environment (RSCy2013), Vol. 8795, p. 879514

Štroner, M., Urban, R., Reindl, T., Seidl, J., Brouček, J., 2020. Evaluation of the georeferencing accuracy of a photogrammetric model using a quadrocopter with onboard GNSS RTK, Sensors, 20(8), 2318.

Taddia, Y., Stecchi, F., Pellegrinelli, A., 2020. Coastal mapping using Dji phantom 4 RTK in post-processing kinematic mode. Drones, 4(2), 1-19.

Zhang, C., 2008. An UAV-based photogrammetric mapping system for road condition assessment. Int. Arch. Photogramm., Remote Sens. Spatial Inf. Sci., XXXVII - Part B5, 627-632.

Zhang, Y., Yuan, X., Li, W., Chen, S., 2017. Automatic power line inspection using UAV images. Remote Sensing, 9(8), 824.

Zhang, H., Aldana-Jague, E., Clapuyt, F., Wilken, F., Vanacker, V., Van Oost, K., 2019. Evaluating the potential of postprocessing kinematic (PPK) georeferencing for UAV-based structure-from-motion (SfM) photogrammetry and surface change detection. Earth Surface Dynamics, 7(3), 807-827. 